\title{
Raman scattering in boron-doped single-crystal diamond used to fabricate Schottky diode detectors
}

\author{
G. Faggio $^{\text {a,* }}$, G. Messina ${ }^{\text {a }}$, S. Santangelo ${ }^{\text {a }}$, G. Prestopino ${ }^{b}$, I. Ciancaglioni $^{\mathrm{b}}$, M. Marinelli ${ }^{\mathrm{b}}$ \\ a Dipartimento di Meccanica e Materiali, Università "Mediterranea" di Reggio Calabria, I-89122 Reggio Calabria, Italy \\ b Dipartimento di Ingegneria Industriale Università di Roma "Tor Vergata" I-00133 Roma, Italy
}

\section{A R T I C L E I N F O}

\section{Article history:}

Received 7 March 2012

Received in revised form

16 June 2012

Accepted 18 June 2012

Available online 23 June 2012

Keywords:

Single crystal CVD diamond

Raman spectroscopy

Schottky diodes

UV detectors

\begin{abstract}
A B S T R A C T
Thanks to its exceptional physical and electronic properties, diamond is an attractive material for electronic devices working at high temperature and in harsh chemical environment. Its use as a semiconducting material for electronics is related to the possibility of doping it in order to control its conductivity. Semiconducting p-type diamond films can be grown when boron is introduced into the film. In this work, boron-doped (B-doped) homoepitaxial diamond films are grown by Microwave Plasma Enhanced Chemical Vapor Deposition. Raman and electrical characterizations are carried out on the films as a function of boron doping level. As the boron content increases, we observe systematic modifications in the Raman spectra of single-crystal diamonds. A significant change in the lineshape of the first-order Raman peak, as well as a wide and structured signal at lower wavenumbers, appears simultaneously in samples grown with higher boron content. A single crystal diamond Schottky diode based on a metal/intrinsic/p-type diamond junction is analysed.
\end{abstract}

(c) 2012 Elsevier Ltd. All rights reserved.

\section{Introduction}

Diamond is an ideal material for the fabrication of compact solid-state ionizing radiation detectors [1]. Visibleblindness, chemical inertness, large heat conductance, high charge mobility and low dark current are the properties that make diamond particularly interesting for the realization of photodetectors $[2,3]$. In addition, due to its low atomic number, diamond is a tissue equivalent material, and thus it can be used to realize dosimeters for radiotherapy [4,5]. Natural diamond, mainly because of lack of standardisation, high cost and inclusion of impurities, is not a feasible proposal as an engineering material. Homoepitaxial growth by Chemical Vapour Deposition (CVD) has enabled the synthesis of high-quality single crystal diamond on low-cost high-pressure high-temperature (HPHT) diamond substrates.
Semiconducting p-type diamond films can be grown when boron is introduced into the film. Heavy boron incorporation induces metallic conduction in diamond. Boron-doped (Bdoped) diamond has attracted considerable attention for its application in the fabrication process of novel diamond based multilayered Schottky diode detectors for X-ray and ultraviolet radiation [1], alpha particles, thermal and fast neutrons [6] and radiation therapy dosimetry [7]. In this paper, a series of B-doped homoepitaxial diamond films have been analyzed by Raman spectroscopy. The measurements have been performed at room temperature on films with different boron contents. The conductivity of the samples has been also measured to evaluate the activation energy as a function of boron content. A new class of detectors with a layered structure based on CVD diamond has been investigated.

\section{Experimental}

* Correspondence to: Dipartimento di Meccanica e Materiali, Facoltà di Ingegneria dell Università "Mediterranea" di Reggio Calabria, Località Feo di Vito, I-89122 Reggio Calabria, Italy. Tel.: + 390965 875485; fax: + 390965875201

E-mail address: gfaggio@unirc.it (G. Faggio).
The B-doped homoepitaxial diamond films used in this study have been grown at the University of Rome 
“Tor Vergata” by Microwave Plasma Enhanced Chemical Vapor Deposition (MWPECVD) on commercial low cost synthetic type Ib (100) HPHT single crystal diamond substrates, $4 \times 4 \times 0.5 \mathrm{~mm}^{3}$ in volume. The samples are coded as $A,-D$ in order of increasing boron content in the grown layers. The four films have been grown at different concentrations of a $100 \mathrm{ppm}$ diborane-hydrogen gas mix added to a methane-hydrogen source gas. The grown samples have been electrically characterized by means of electrical resistance vs. temperature measurements. The measurements have been performed by placing in turn the B-doped diamond samples into a temperature controlled furnace and the resistance vs. temperature data have been recorded during spontaneous cooling to room temperature, after a maximum temperature of about $500{ }^{\circ} \mathrm{C}$ has been reached. A Keithley 2000 multimeter has been used for a 2-wire resistance measurement and a Keithley 2001 multimeter has been connected to a type-S platinum/rhodium thermocouple for temperature measurement.

The Raman scattering measurements have been carried out at room temperature with an Instrument S.A. Ramanor U1000 double monochromator, equipped with a microscope Olympus BX40 for micro-Raman sampling and with an electrically cooled Hamamatsu R943-02 photomultiplier for photon-counting detection or with a CCD detector. The $514.5 \mathrm{~nm}(2.41 \mathrm{eV})$ line of an $\mathrm{Ar}^{+}$ion laser (Coherent Innova 70) has been used to excite Raman scattering. Using a X100 objective, the laser beam has been focused to a diameter of approximately $1 \mu \mathrm{m}$. A depth resolution of approximately $4 \mu \mathrm{m}$ has been obtained with a confocal aperture of $200 \mu \mathrm{m}$. The resolution of the double monochromator is approximately $0.15 \mathrm{~cm}^{-1}$. After normalisation, all the spectra collected are analysed using a commercially available spectroscopic analysis software package. Lorentzian bands, superimposed to a constant background, are used to fit the spectra. The wavenumber position, width (FWHM) and intensity of the bands are chosen by a leastsquare best-fit method.

A single crystal diamond Schottky diode detector based on a metal/intrinsic/p-type diamond junction has been produced. The multilayered structure is obtained by a wellestablished two-step Chemical Vapor Deposition process. A heavily boron doped CVD diamond film, with a boron concentration of approximately $0.5 \times 10^{20} \mathrm{~cm}^{-3}$, is deposited, at first, on a low cost commercial synthetic single crystal HPHT diamond substrate. A nominally intrinsic diamond film, which acts as a sensing layer of the device, is then homoepitaxially deposited on the doped one. This second step is performed in a completely separate CVD reactor in order to avoid unintentional boron contamination of the intrinsic layer. A circular aluminum (Al) electrode, about $3 \mathrm{~mm}$ in diameter, is thermally evaporated on the diamond surface, forming a rectifying Schottky junction with the intrinsic diamond. An annealed silver $(\mathrm{Ag})$ paint is utilized in order to provide an ohmic contact to the boron doped layer. A sketch of the multilayered device structure and of the electrical connections is shown in Fig. 1. The highly conductive boron doped diamond film acts as backing contact. The sensing volume of the detector is the depletion region that from the Schottky metal-diamond interface extends into the intrinsic diamond layer [1]. Such a sandwich

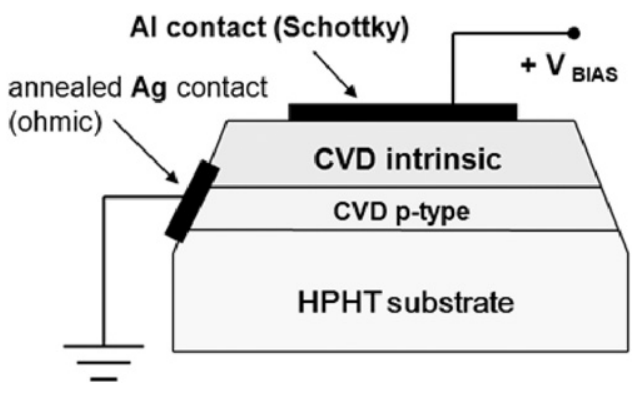

Fig. 1. Scheme of the typical device configuration of synthetic single crystal diamond detectors fabricated at the University of Rome "Tor vergata”.

geometry has been adopted to avoid any contribution to the response of the high quality, detector grade intrinsic CVD sensing layer coming from the HPHT diamond substrate, with no need to mechanically remove it.

\section{Results and discussion}

\subsection{Electrical characterization of B-doped diamond films}

Boron doping makes the diamond films p-type semiconductors [8]. At low boron concentration $\left(\sim 10^{18} \mathrm{~cm}^{-3}\right)$ an electrical conductivity on the order of $10^{-2} \Omega^{-1} \mathrm{~cm}^{-1}$ has been measured at room temperature in homoepitaxial diamond films $[9,10]$. The acceptor level lies rather deep into the band-gap with an activation energy of about $0.37 \mathrm{eV}$ from the valence band [8-10]. When the boron concentration exceeds the value of $3 \times 10^{20} \mathrm{~cm}^{-3}$, an impurity band is formed and a metallic-like conductivity is observed [11,12]. The change in conductivity of diamond film from p-type semiconductor to metallic diamond is highlighted by the decrease towards zero of the boron activation energy $[9,10]$.

In order to evaluate the activation energy, the conductivity of the samples has been measured between room temperature and $700 \mathrm{~K}$ (Fig. 2). Activation energies, reported in Table 1, have been obtained from the best fit of the Arrhenius plot of the measured data in the temperature range 500-700 K. From the activation energy values, the acceptor densities $N_{a}$ have been roughly estimated by the model developed by Pearson and Bardeen [13] for silicon:

$E_{a}=E_{0-} \alpha N_{a}^{1 / 3}$ where $E_{0}$ is the ionization energy of an isolated dopant and $\alpha$ is a material dependent constant, whose value for diamond is about $6.7 \times 10^{-8} \mathrm{eV} \mathrm{cm}$. From this model, for activation energies ranging between 0.23 and $0.04 \mathrm{eV}$, boron concentrations from $\sim 10^{18}$ to $\sim 10^{20} \mathrm{~cm}^{-3}$ are evaluated. Such values are approximate, due to the low accuracy in the activation energy measurements and the simplified model used for their determination. The decrease of the values of the activation energy with increasing boron content highlights the gradual change of conductivity of the samples from p-type semiconductor to metallic diamond.

\subsection{Raman characterization of B-doped diamond films}

Raman spectroscopy is a nondestructive and powerful technique to investigate the structure and the properties 


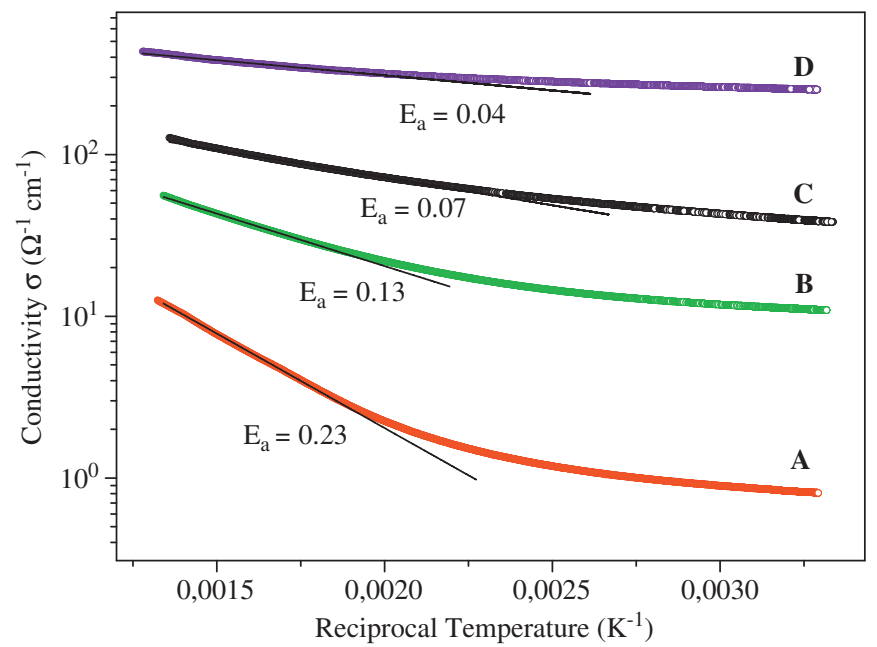

Fig. 2. Conductivity of B-doped homoepitaxial diamond films as function of reciprocal temperature.

Table 1

Frequency position, $\omega$ and width, $\Gamma$, of the diamond Raman peaks of the B-doped homoepitaxial diamond films. The activation energy, Ea, and the boron concentrations, $\mathrm{Na}$, of the samples are also reported.

\begin{tabular}{lcccl}
\hline Sample & $\omega\left(\mathrm{cm}^{-1}\right)$ & $\Gamma\left(\mathrm{cm}^{-1}\right)$ & $E_{a}(\mathrm{eV})$ & $N_{a}\left(\mathrm{~cm}^{-3}\right)$ \\
\hline Substrate & 1332.1 & 2.2 & - & - \\
A & 1332.3 & 2.3 & 0.23 & $8.7 \times 10^{18}$ \\
B & 1331.4 & 3.8 & 0.13 & $4.5 \times 10^{1}$ \\
C & 1331.0 & 7.3 & 0.07 & $8.8 \times 10^{19}$ \\
D & 1331.6 & 13.7 & 0.04 & $1.2 \times 10^{20}$ \\
\hline
\end{tabular}

of diamond. For a high quality single crystal diamond, the Raman spectrum is dominated by a single sharp line at $1332 \mathrm{~cm}^{-1}$, whose full-width at half-maximum (FWHM) is approximately $2 \mathrm{~cm}^{-1}$, which corresponds to inelastic scattering of the laser light by the triply degenerate optical phonon at the Brillouin zone centre. In B-doped diamond films, both polycrystalline and homoepitaxial, the diamond Raman line displays increasing asymmetry and shifts to lower frequency with increasing $B$ concentration [12,14,15]. The Raman spectrum of heavily B-doped diamond exhibits not only a pronounced asymmetry and downshift of the " $1332 \mathrm{~cm}^{-1}$ " Raman line, but also two broad bands centred at approximately $500 \mathrm{~cm}^{-1}$ and $1225 \mathrm{~cm}^{-1}$ and another one of small intensity centred at approximately $1000 \mathrm{~cm}^{-1}[14,16,17]$.

Fig. 3 shows the Raman spectra in the wave number region of $400-1600 \mathrm{~cm}^{-1}$ measured on the surface of the samples synthesized at various B-doping levels. At low boron content, the spectrum of the sample $A$ is very similar to that of the HPHT single crystal diamond substrate, exhibiting only the characteristic peak at $1332 \mathrm{~cm}^{-1}$. Two wide bands at approximately $500 \mathrm{~cm}^{-1}$ and $1225 \mathrm{~cm}^{-1}$ and another one of low intensity at approximately $1000 \mathrm{~cm}^{-1}$ are added on the low frequency side of the spectrum of the samples grown with higher boron content (samples B-D). These bands are commonly observed in the Raman spectra of polycrystalline and homoepitaxial heavily boron-doped diamond films [14,16], although their origin is still a matter of controversy. The phonon density of states of

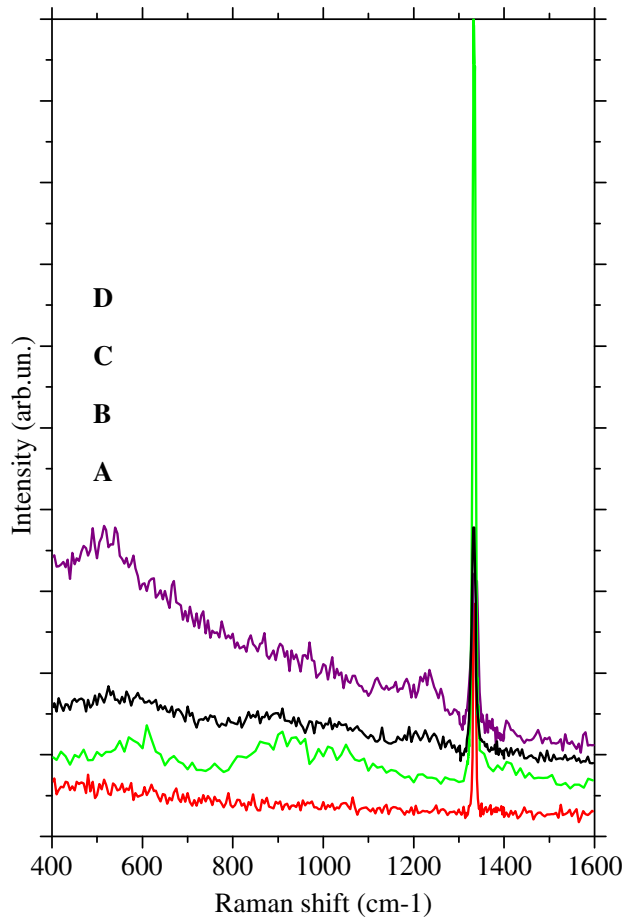

Fig. 3. Raman spectra of the B-doped homoepitaxial diamond films at different boron concentrations.

diamond presents a main asymmetric maximum around $1250 \mathrm{~cm}^{-1}$ and secondary maximum around $550 \mathrm{~cm}^{-1}$ and hence their origin has been attributed to the relaxation of selection rules induced by the high boron content [18]. Later, Bernard et al. [16] have proposed that the $500 \mathrm{~cm}^{-1}$ band originates from local vibration modes of boron pairs.

Fig. 4 shows the line shape of the first-order Raman peak for the samples investigated. The spectra have been recorded by carefully focusing the laser spot at the sample surface. As the boron content increases, the thickness of the grown films gradually decreases and then it becomes increasingly difficult to exclude the contribution of the 
substrate. With the exception of the sample with lower boron content (sample A), all the peaks exhibit a downshift and a broadening with respect to the Raman peak of the undoped substrate. The frequency position, $\omega$, and the full width at half maximum, $\Gamma$, of the peaks are reported in Table 1 . The asymmetric broadening and the downshift of the one-phonon Raman line of B-doped diamond observed both in polycrystalline and in homoepitaxial samples [14-16,19], have been explained as the result of a Fano-type discrete-continuum interaction between the discrete zone-centre phonon state and the continuum of

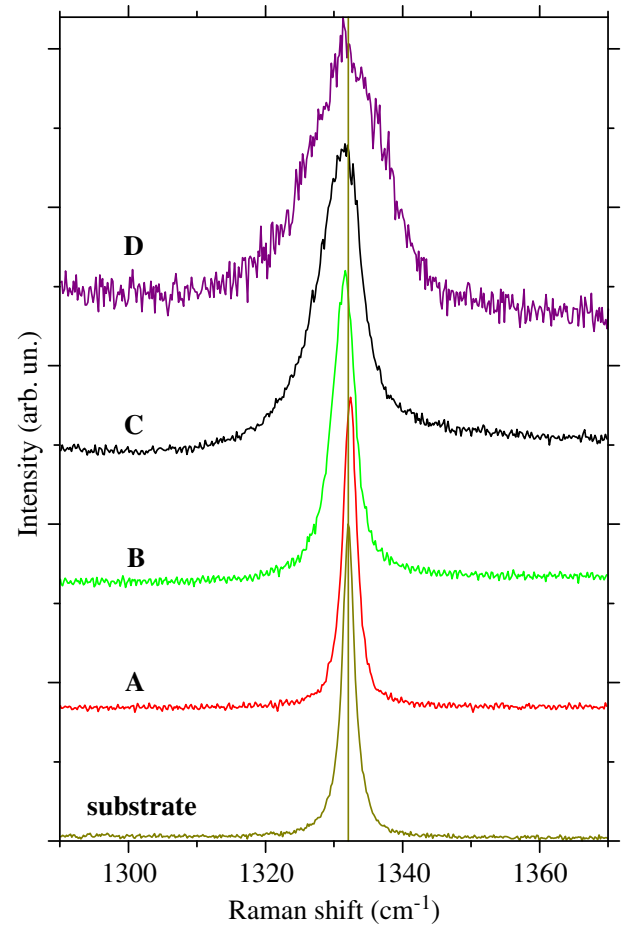

Fig. 4. First-order Raman peaks of the B-doped homoepitaxial diamond films at different boron concentrations. the electronic states induced by the presence of the dopant $[15,17,19,20]$. Moreover, dopant incorporation at substitutional or interstitial sites can produce stress that generally depends on the doping level. Therefore, it is possible that the changes observed in first-order Raman peak of the more doped samples (Fig. 4) reflect both a Fano-type interaction and the presence of tensile stress. At the doping levels used in this work, lower than the critical threshold of $\sim 3 \times 10^{20} \mathrm{~cm}^{-3}$ [21] to clearly observe a strong Fano deformation of the Lorentzian Raman line, it is difficult to distinguish between the two mechanisms [22,23].

Although boron concentration of our samples is not high enough to clearly show the Fano effect, the comparison between the results of Raman analysis and electrical characterization shows the existence of a correlation between the full width at half maximum, $\Gamma$, of the diamond Raman peak and the activation energy, $E_{a}$, deduced from conductivity measurements. An interesting linear correlation is observed in the log-log plot of $\Gamma$ vs. $E_{a}$ (Fig. 5). Although further measurements are necessary to check the validity range of the shown correlation, this finding suggests the possibility of quickly evaluating the activation energy $E_{a}$ of boron-doped samples by simply measuring the linewidth of their Raman peak, rather than by time consuming electrical measurements. Then, by using the Pearson-Barden model, the dopant concentration could be evaluated.

\section{Diamond based multilayered detectors}

Detectors based on single crystal CVD diamond in a multilayered metal/intrinsic/p-type configuration have been recently produced at the laboratories of the University of Rome "Tor Vergata". Such detectors have been successfully utilized for the fabrication of high performance Schottky barrier (SB) particle detectors (alpha particles, neutrons) [6], photon detectors (vacuum UV, soft $X$ rays) [1] and radiation therapy dosimeters [7].

The working principle of diamond based multilayer detectors is based on the existence of a built-in voltage

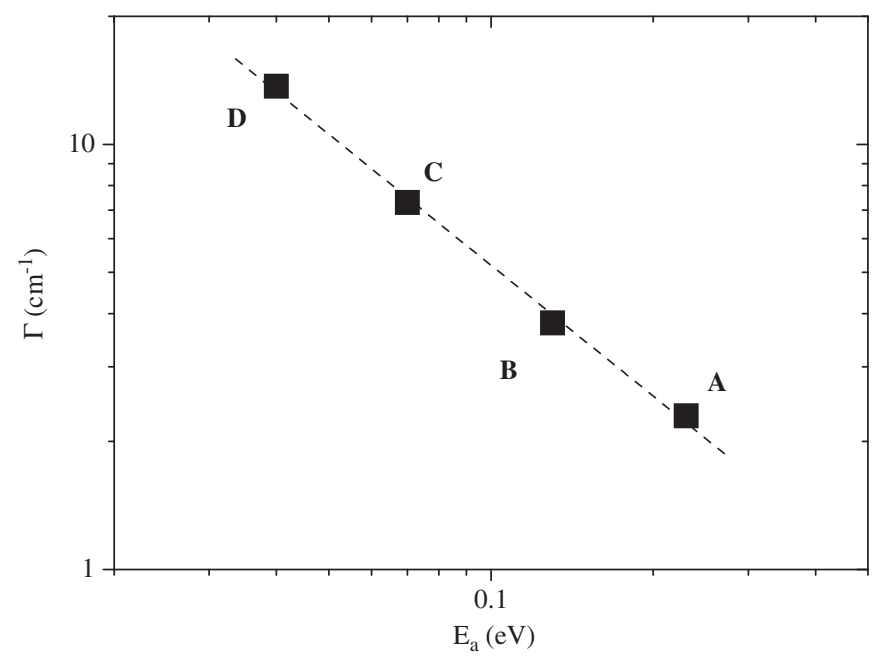

Fig. 5. Full width at half maximum, $\Gamma$, of the diamond Raman peak vs. the activation energy, $E_{a}$. 
barrier located between the $\mathrm{Al}$ metal contact and the nominally intrinsic layer, and the device acts thus as a Schottky barrier diode. Such a detector is able to operate both in photoconductive mode, by reverse-biasing $\left(V_{B I A S}>0\right.$ in Fig. 1) the Schottky junction, and in photovoltaic mode $\left(V_{B I A S}=0\right)$, by using the built-in potential of the Schottky barrier. The former configuration is mainly used for particle detection, achieving highly reproducible results in terms of $100 \%$ charge collection efficiency and high energy resolution; the latter is used for photon detection and radiation therapy dosimetry. Such devices cannot be operated in the forward bias regime $\left(V_{\text {BIAS }}<0\right.$ in Fig. 1) since leakage currents rapidly increase due to hole injection from the borondoped single crystal diamond (SCD) layer.

Fig. 6(a) shows the current-voltage characteristic of a typical UV diamond based multilayered Schottky photodiode. Dark current and photocurrent have been measured vs. applied bias voltage at room temperature by a Keithley 6517A electrometer, using its internal voltage source. The dark current is lower than $10^{-13}$ A for the tested reverse bias voltages $\left(0 \mathrm{~V} \leq V_{B I A S} \leq 10 \mathrm{~V}\right)$ and suddenly increases under forward bias $\left(V_{B I A S}<0 \mathrm{~V}\right)$. A very good rectification ratio of about $10^{8}$ can be observed at $\pm 3 \mathrm{~V}$. Under irradiation with UV light from a DC gas discharge He-Ne lamp, the diamond detector clearly shows a photocurrent response at zero voltage bias, due to the built in potential at the Schottky barrier (device operated photovoltaic mode). The photocurrent is almost constant with increasing positive voltage (photoconductive mode), while the dark current increases by about two orders of magnitude. Remarkably, as shown

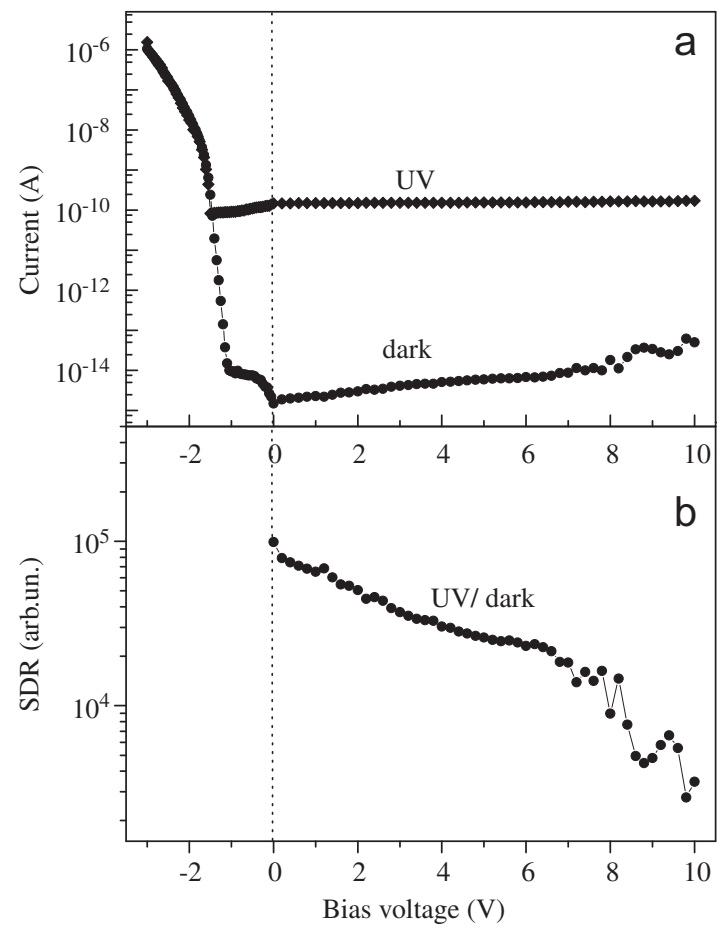

Fig. 6. Current-voltage characteristic in dark and in UV light of a SCD based Schottky barrier photodetector (a). The signal-to-dark ratio (SDR) vs. the applied bias voltage is also shown (b). in Fig. 6(b), the best signal-to-dark ratio (SDR) is obtained at zero bias voltage, which is chosen as the typical, best performance, operating condition for such detectors.

\section{Conclusions}

Boron-doped MWPECVD single-crystal diamonds have been grown at increasing boron content. Raman and electrical characterizations have been carried out on films as a function of boron doping level. Activation energies ranging between 0.23 and $0.04 \mathrm{eV}$ have been evaluated by conductivity vs. temperature measurements. From these values, boron concentrations from $\sim 10^{18}$ to $\sim 10^{20} \mathrm{~cm}^{-3}$ have been estimated. The Raman spectrum of the sample grown with low boron content exhibits only the characteristic diamond peak at $1332 \mathrm{~cm}^{-1}$. With increasing doping level, the first-order Raman peak appears broadened and shifted to lower wavenumbers with respect to the Raman peak of the undoped substrate. In the same samples, two wide bands at approximately $500 \mathrm{~cm}^{-1}$ and $1225 \mathrm{~cm}^{-1}$ and another one of low intensity at approximately $1000 \mathrm{~cm}^{-1}$ have been observed on the low frequency side of the spectrum.

Finally, the electrical properties of a typical UV detector with a structure that acts as a metal/intrinsic/p-doped diamond photovoltaic Schottky diode have been investigated.

\section{References}

[1] Almaviva S, Marinelli M, Milani E, Prestopino G, Tucciarone A Verona $C$, et al. Chemical vapor deposition diamond based multilayered radiation detector: physical analysis of detection properties. J Appl Phys 2010;107:014511.

[2] Field JE. Properties of diamond. London: Academic Press; 1979.

[3] Prins J. Applications of diamond films in electronics. In: Paoletti A, Tucciarone A, editors. The physics of diamond. Amsterdam: IOS Press; 1997. p. 411-75.

[4] Almaviva S, Marinelli M, Milani E, Tucciarone A, Verona RG Consorti R, et al. Synthetic single crystal diamond diodes for radiotherapy dosimetry. Nucl Instrum Methods Phys Res A 2008;594:273.

[5] Tromson D, Descamps C, Tranchant N, Bergonzo P, Nesladek M, Isambert A. Investigations of high mobility single crystal chemical vapor deposition diamond for radiotherapy photon beam monitoring. J Appl Phys 2008;103:054512.

[6] Marinelli M, Milani E, Prestopino G, Scoccia M, Tucciarone A Verona-Rinati $\mathrm{G}$, et al. High performance LiF-diamond therma neutron detectors. Appl Phys Lett 2006;89:143509.

[7] Almaviva S, Ciancaglioni I, Consorti R, De Notaristefani F, Manfredotti C, Marinelli M, et al. Synthetic single crystal diamond dosimeters for Intensity Modulated Radiation Therapy applications. Nucl Instr Meth A 2009;608:191.

[8] Collins AT, Williams ASW. The nature of the acceptor centre in semiconducting diamond. J Phys C: Solid State Phys 1971;4:1789.

[9] Thonke K. The boro acceptor in diamond. Semicond Sci Technol 2003; 18:S20.

[10] Borst $\mathrm{TH}$, Weis O. Electrical characterization of homoepitaxial diamond films doped with $\mathrm{B}$. Li, and Na during crystal growth. Diamond Relat Mater 1955;4:948.

[11] Deneuville A, Baron C, Ghodbane S, Agnes C. Highly and heavily boron doped diamond films. Diamond Relat Mater 2007;16:915.

[12] Ager III JW, Walukiewicz W, McCluskey M, Plano MA, Landstrass MI. Fano interference of the Raman phonon in heavily boron-doped diamond films grown by chemical vapor deposition. Appl Phys Lett 1994;616:66.

[13] Pearson GL, Bardeen J. Electrical properties of pure silicon and silicon alloys containing boron and phosphorus. Phys Rev 1949;75: 865.

[14] Pruvost F, Bustarret E, Deneuville A. Characteristics of homoepitaxial heavily boron-doped diamond films from their Raman spectra. Diamond Rel Mater 2000;9:295. 
[15] Prouvost F, Deneuville A. Analysis of the Fano in diamond. Diamond Relat Mater 2001;10:531.

[16] Bernard M, Baron C, Deneuville A. About the origin of the low wave number structures of the Raman spectra of heavily boron doped diamond films. Diamond Rel Mater 2004;13:896.

[17] Roy M, Dua AK, Nuwad J, Girija KG, Tyagi AK, Kulshreshtha SK. Controlled in situ boron doping of diamond thin films using solution phase. J Appl Phys 2006;100:124506.

[18] Gheeraert E, Gonon P, Deneuville A, Abello L, Lucazeau G. Effect of boron incorporation on the quality of MPCVD diamond films. Diamond Rel Mater 1993;2:742.

[19] Prawer S, Nemanich RJ. Raman spectroscopy of diamond and doped diamond. Philos Trans R Soc London A 2004;362:2537.
[20] Cerdeira F, Fjeldly TA, Cardona M. Effect of free carriers on zonecenter vibrational modes in heavily doped p-type Si. II. optical modes. Phys Rev B 1973;8:4734.

[21] Brunet F, Germi P, Pernet M, Deneuville A, Gheeraert E, Laugier F, et al. Microstructure evolution of boron doped homoepitaxial diamond films. J Appl Phys 1998;83:181.

[22] Wang WL, Polo MC, Sanchez G, Cifre J, Esteve J. Internal stress and strain in heavily boron-doped diamond films grown by microwave plasma and hot filament chemical vapor deposition. J Appl Phys 1996;80:1846.

[23] Li H, Zhang T, Li L, Lü X, Li B, Jin Z, et al. Investigation on crystaline structure, born distribution, and residual stresses in freestanding boron-doped CVD diamond films. J Crystal Growth 2010(312):1986. 